\title{
METHOD OF FORMING RECOMMENDATIONS USING TEMPORAL CONSTRAINTS IN A SITUATION OF CYCLIC COLD START OF THE RECOMMENDER SYSTEM
}

\author{
Serhii Chalyi \\ Department of Information Control Systems \\ Kharkiv National University of Radio Electronics \\ 14 Nauka ave., Kharkiv, Ukraine, 61166 \\ serhii.chalyi@nure.ua \\ Volodymyr Leshchynskyi \\ Department of Software Engineering \\ Kharkiv National University of Radio Electronics \\ 14 Nauka ave., Kharkiv, Ukraine, 61166 \\ volodymyr.leshchynskyi@nure.ua \\ Irina Leshchynska \\ Department of Software Engineering \\ Kharkiv National University of Radio Electronics \\ 14 Nauka ave., Kharkiv, Ukraine, 61166 \\ iryna.leshchynska@nure.ua
}

\begin{abstract}
The problem of the formation of the recommended list of items in the situation of cyclic cold start of the recommendation system is considered. This problem occurs when building recommendations for occasional users. The interests of such consumers change significantly over time. These users are considered "cold" when accessing the recommendation system. A method for building recommendations in a cyclical cold start situation using temporal constraints is proposed. Temporal constraints are formed on the basis of the selection of repetitive pairs of actions for choosing the same objects at a given level of time granulation. Input data is represented by a set of user choice records. For each entry, a time stamp is indicated. The method includes the phases of the formation of temporal constraints, the addition of source data using these constraints, as well as the formation of recommendations using the collaborative filtering algorithm. The proposed method makes it possible, with the help of temporal constraints, to improve the accuracy of recommendations for "cold" users with periodic changes in their interests.
\end{abstract}

Keywords: recommendation system, temporal constraints, personalization of recommendations, area under the curve.

DOI: $10.21303 / 2461-4262.2019 .00952$

\section{Introduction}

Recommendation systems are used to support consumer choice in the field of e-commerce, for example, in online stores, hotel booking systems, streaming video sales systems. Such systems are designed to predict a set of items that may be interesting to a specific user in the absence of information about the choice of specified items by this user. In making recommendations data on the choice of other users, as well as on the characteristics of selected items are used [1].

In the process of building such systems, a cold start problem usually arises due to the incompleteness of data on new users [2]. In the case of a new user or a new product, the latter are defined as "cold". This means that information on these products is not enough to form accurate recommendations. The recommendation system can offer the necessary goods only after the user selects them on its own initiative. Such users are no longer "cold" and information about them can be used to support consumer choice. A cold start is an important problem in the recommendation system for occasional users who visit the corresponding site periodically, at long intervals, for example, to buy household appliances, books, and holiday travel [3]. Solving the problem of a cyclic cold start is associated with difficulties due to the lack of dependency information affecting consumer choice. Such implicit dependencies can be obtained based on the analysis of the sequence of user actions [4]. 
To solve the cold start problem, hybrid methods are used based on the complement of collaborative filtering by analyzing context data [5]. Also demographic data are used [6]. To account for cyclical changes in the interests of users, their temporal characteristics are used. Research in this direction uses two approaches: based on the user model and based on data filtering. In the first case, models for one and several users are used. In [7], a model of seasonal changes in the interests of the user was proposed, which is trained using gradient descent. The model of user preferences in the form of a temporal graph, which uses a random search when generating recommendations, is considered in [8]. The neural network model of multiple user behavior was proposed in [9]. In accordance with the second approach, using partial filtering of input data removes information that does not reflect the current interests of users. For filtering, a model based on a multilayer graph [10] is used, as well as the principles of self-learning [11] and active learning [12]. Approaches based on the model can more accurately describe the behavior of the user. Approaches based on input filtering are easily integrated with existing methods of building recommendations.

However, in general, the considered approaches are not focused on solving the problem of a cyclical cold start, since the latter is also characterized by a change in consumer interests and incomplete data on the choice of these consumers. To solve this problem, it is advisable to modify the input data based on the temporal model of the cycle of selection of known users, and then formulate recommendations for the new user. Therefore, the main idea of the research consists in combining the user behavior model, as well as modifying the input data when generating recommendations in a cyclical cold start situation.

\section{Method of forming recommendations in situation of cyclic cold start using temporal con- straints on input data}

The developed method is intended for building recommendations in the case when input information, which is used to support the choice of the current consumer, is absent or irrelevant. Such information is usually absent for a new user and becomes irrelevant for irregular users when their interests change over time.

The method complements the input data set, which is used to build recommendations, before performing collaborative filtering. Supplement data is based on the use of temporal dependencies. Temporal dependencies have the form of weighted rules [13]. The weights of these rules are determined based on a random search [14]. Temporal dependencies in recommendation systems characterize selection sequences by well-known consumers of products that may be of interest to the current user. It is necessary to select and order the sequence of events (choice) for their formation, taking into account the context of this choice. A general approach to the selection of the initial subsets of events was proposed in [15].

The purpose of supplementing the input data is to set restrictions for the new, "cold" user in the form of permissible temporal dependencies of the choice of goods or services.

As a source of data in the recommendation systems, a matrix $E$ of choice (for example, purchases) of the consumer is usually used. This matrix is sparse, that is, only a small part of the elements of the matrix is non-zero and displays the connection of users with items.

Each element $e_{k j}$ of this matrix characterizes the choice of a consumer $u_{k}$ from a set of consumers $U$ of an item (product, service) $i_{j}$. Each non-zero element $e_{k j}$ contains either the value of the product rating $i_{j}$ or the number of units of this product purchased by the consumer $u_{k}$. For simplicity, let's assume that the element $e_{k j}$ contains the number of purchased items.

Traditionally, such a matrix summarizes information about each choice (purchase) $e_{k j}^{n}$ of items $i_{j}$ by the user $u_{k}$.

This selection is performed sequentially over a specified time interval $\left[\tau_{1}, \tau_{N}\right]$ :

$$
\forall e_{k j}^{\mathrm{n}} \exists \tau_{n}: \tau_{1} \leq \tau_{n} \leq \tau_{N}, \tau_{n-1}<\tau_{n}
$$

This means that each element of the matrix $E$ combines a set of consumer choices (purchases, ratings) ordered in time: 


$$
e_{k j}=\left\langle e_{k j}^{1}, e_{k j}^{2}, \ldots, e_{k j}^{n}, \ldots, e_{k j}^{\mathrm{N}}\right\rangle
$$

When several users select several $\left[\tau_{1}, \tau_{N}\right]$ products in an interval, let's obtain the sequence of selecting $E_{1}^{\mathrm{N}}$ :

$$
E_{1}^{\mathrm{N}}=\left\langle e_{k j}^{1}, e_{l j}^{2}, \ldots, e_{k m}^{n}, e_{l m}^{n+1}, \ldots\right\rangle
$$

This sequence contains a pattern of user behavior linking the purchase of goods $i_{j}$ and $i_{m}$. Indeed, users $u_{k}$ and $u_{l}$ in the moments of time $\tau^{1}$ and $\tau^{2}$ chose the same product $i_{j}$. After some time, in moments $\tau^{n}$ and $\tau^{n+1}$ the same product $i_{m}$ was also selected. Thus, the sequence (3) reflects the cyclical behavior of the users.

Such dependencies are not used in the traditional construction of recommendations, but are essential when cyclically cold start, since information about purchases periodically becomes irrelevant. As a result, when user preferences change, it is necessary to collect and analyze data on user needs.

An example of user selection cycles is shown in Fig. 1. This example shows the sequence of user selection in the six weeks preceding the appearance of a new user. At the moment of time $\tau_{11}$ a new, "cold" user $u_{6}^{\text {Cold }}$ chooses an item $i_{3}$.

The selection sequence is shown in Fig. 1 as follows. At the time point $\tau_{1}$, the buyer $u_{1}$ chose the item $i_{1}$. At the time $\tau_{2}$, the buyer $u_{4}$ chose the item $i_{4}$, etc.

Analysis of this sequence shows that the $u_{1}$ user's choice of a pair of items $\left(i_{1}, i_{4}\right)$ is repeated twice in about 2 weeks and does not change when other interests of users change.

Therefore, it is advisable to consider this pair as a temporal limitation: after choosing $i_{1}$, a user has to choose $i_{4}$.

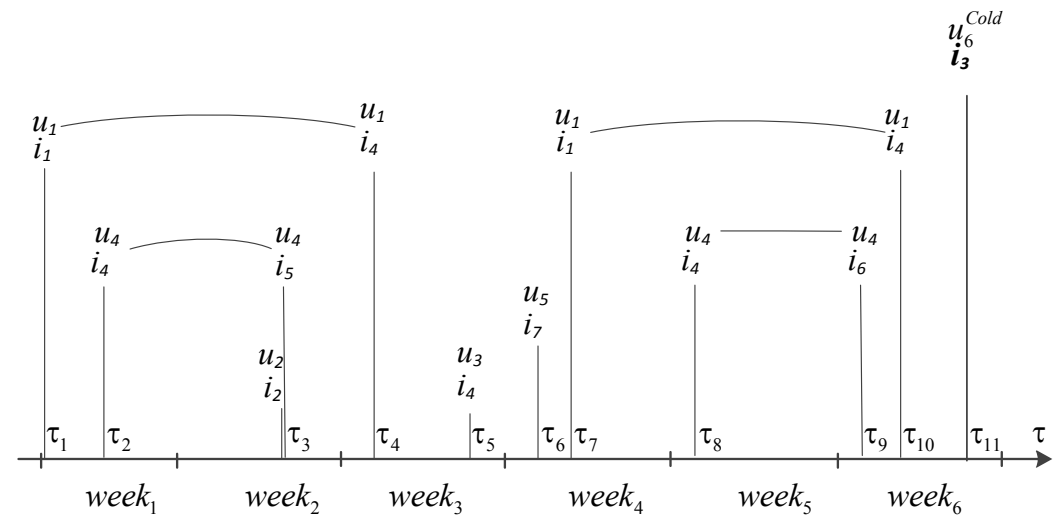

Fig. 1. The sequence of user selection

The buyer $u_{4}$, after selecting an item $i_{4}$ after about a week in one case, chose the product $i_{5}$, and in the other $-i_{6}$. Such dependencies should be considered as rules with the same weight, which corresponds to the probability of their realization. for them.

Other buyers have chosen only one product; therefore there are no temporal dependencies

Thus, there are 3 temporal dependencies in the given example: constraints $\left(i_{1}, i_{4}\right)$ and rules $\left(i_{4}, i_{5}\right)$ and $\left(i_{4}, i_{6}\right)$.

These constraints and rules can be ad hoc or typical for a given domain, or for these items. If the frequency of occurrence of these constraints or rules in the input data exceeds a certain threshold value, then the dependencies between the specified objects should be considered typical. The threshold value depends on the domain. Therefore it should be chosen experimentally. Typical rules apply when specifying input to a new user.

In [13], several types of temporal rules are presented that are focused on decision-making support in process management. Such rules must be adapted to the given features of support rec- 
ommendations. In this paper, we use a modified rule of the type "Next", which links two successive purchases at a given level of time granulation.

The temporal rule of a sequential selection of items in the recommendation system sets a pair of consecutive events for the selection of goods or services $e_{k j}$ and $e_{k m}$ by the user $u_{k}$ at times $\tau_{n}$ and $\tau_{l}$ at a given level of time granulation $\Delta \tau$. This rule is set using the temporal operator $X(\mathrm{Next})[13]$ :

$$
\exists u_{k}:\left(e_{k j}, \tau_{n}\right) X\left(e_{k m}, \tau_{l}\right)|| \tau_{n}-\tau_{l} \mid \approx \Delta \tau
$$

In accordance with rule (4), there are no intermediate purchases at the time level $\Delta \tau$.

When selecting objects in the recommendation system sequentially temporal restriction sets a pair of consecutive events for which a strict order is set at a given level of time granulation $\Delta \tau$ :

$$
\exists j, m: \forall k\left(e_{k j}, \tau_{n}\right) X\left(e_{k m}, \tau_{l}\right)|| \tau_{n}-\tau_{l} \mid \approx \Delta \tau
$$

By virtue of the condition $\forall k$, the constraint (5) will continue to be presented without the user's index $k$, as a pair $\left(e_{j}, e_{m}\right)$.

Restrictions (5) can be used to supplement information about a new user. Such dependences simulate a change in user interests and allow you to modify the source data in a cyclical cold start situation.

The formation and use of temporal limitations in building recommendations is carried out within the framework of the developed method. The method uses temporal dependencies for a given level of time granulation.

As input data are used: sales log containing a set of triples $L=\left\{\left(u_{k}, i_{j}, \tau_{n}\right)\right\}$; time interval for input data $\left[\tau_{1}, \tau_{N}\right]$; level of time granulation $\Delta \tau$; threshold number $\alpha$ of repetitions of the rule. The last parameter is used when choosing temporal constraints.

The method includes the following steps.

Stage 1. Selection of a subset of sales log records for a given time interval $\left[\tau_{1}, \tau_{N}\right]$. This interval depends on the subject area. For example, for the task of booking hotels such an interval can be a year, sales of clothes - a season, and so on. The selection condition at this stage is $\tau_{n} \in\left[\tau_{1}, \tau_{N}\right]$.

Stage 2. Generalization of selection events for a given time granulation $\Delta \tau$. At this stage, the values $\tau_{n}$ are summarized according to the level of detail - up to hours, days, etc.

Stage 3. Formation of pairs $\left(e_{j}, e_{m}\right)$ of sequential selection (purchases) of users at a given level of time granulation. The result of this stage is a set $R$ that includes the pairs $\left(e_{j}, e_{m}\right)$ and the number of repetitions of these pairs $n_{j m}$ on the set of input data:

$$
R=\left\{\left(e_{j}, e_{m}, n_{j m}\right): e_{j} X e_{m}, n_{j m} \geq 2\right\}
$$

Stage 4. Formation of temporal constraints $C$ for the choice of users:

$$
C=\left\{\left(e_{j}, e_{m}\right): \forall j\left|\left\{e_{j}\right\}\right|=\left|\left\{e_{j}, e_{m}\right\}\right|=n_{j m}\right\} .
$$

The restriction (7) means that after selecting an item $i_{j}$, users must select an item $i_{m}$. The number of cases of choice is $n_{j m}$.

Stage 5. Selection of temporal restrictions for users.

At this stage, a subset of constraints is selected for each set $\mathrm{C}$, for which $n_{j m}>\alpha$ :

$$
C_{\alpha}=\left\{\left(e_{j}, e_{m}\right): n_{j m}>\alpha\right\}
$$

The result of this phase is a set of constraints for all users.

Stage 6. Supplementing the input data $L$ with constraint records according to (8) for new, "cold" users.

The resulting $\log L^{\text {Cold }}$ has the following form: 


$$
L^{\text {Cold }}=L \bigcup\left\{\left(u_{k}^{\text {Cold }}, i_{m}, \tau_{l}\right): n_{j m}>\alpha\right\}
$$

Stage 7. Building recommendations using traditional methods, in particular collaborative filtering [16]. The result of this stage is a list of recommended items for a new user. The list takes into account cycles of changes in the interests of known users.

\section{Experimental results}

Consider the implementation of the method on the example of the sequence of user selection, shown in Fig. 1. The sales lot $\mathrm{L}$ is presented in this figure in a graphical form. The level of time granulation $\Delta \tau$ is one week. The base time interval $\left[\tau_{1}, \tau_{10}\right]$ covers all 6 weeks. The threshold number of repetitions $\alpha$ is 1 . The original data are supplemented for the new user $u_{6}^{\text {cold }}$.

The phased implementation of the method is presented in Table 1.

Table 1

The results of the method in stages

\begin{tabular}{|c|c|c|}
\hline Stage number & Stage results & Comments \\
\hline 1 & $L$ & $\begin{array}{l}\text { The interval }\left[\tau_{1}, \tau_{10}\right] \text { covers all input data. Therefore, the } \log L \text { is transferred to the } \\
\text { second stage in full }\end{array}$ \\
\hline 2 & $\begin{array}{c}\tau_{1}, \tau_{2} \in \text { wee } k_{1}, \quad \tau_{3} \in \text { week }_{2}, \\
\tau_{4}, \tau_{5} \in \text { week }_{3}, \\
\tau_{6}, \tau_{7} \in \text { week }_{4}, \\
\tau_{8} \in \text { wee } k_{5}, \\
\tau_{9}, \tau_{10} \in \text { wee }_{6}\end{array}$ & $\begin{array}{l}\text { The input data instead of the exact time stamps } \tau_{1}-\tau_{10} \text { get values with a detail of up } \\
\text { to one week }\end{array}$ \\
\hline 3 & $R=\left\{\begin{array}{l}\left(e_{1}, e_{4}, 2\right),\left(e_{4}, e_{5}, 1\right), \\
\left(e_{4}, e_{6}, 1\right)\end{array}\right\}$ & A set of temporal dependencies are formed for all known users \\
\hline 4 & $C=\left\{\left(e_{1}, e_{4}\right)\right\}$ & A set of constraints $C$ is formed from a single repeating element \\
\hline 5 & $\left(e_{1}, e_{4}\right)$ & The constraint satisfies the condition $n_{14}>\alpha$ \\
\hline 6 & $L \cup\left(u_{1}, i_{4}, \tau_{11}\right)$ & The input data of the new user $u_{6}^{\text {Cold }}$ is supplemented with the constraints \\
\hline
\end{tabular}

The restriction obtained in step 5 makes it possible to establish a potential connection between the choice of the user $u_{6}^{\text {Cold }}$ and the known preferences of other users. This connection is depicted by the horizontal arrow in the oval in Fig. 2.

In Fig. 2 input data are presented in the traditional form, without taking into account time stamps. Information about the new user $u_{6}^{\text {Cold }}$ includes the selection of item $i_{1}$. This choice does not allow comparing its interests with the choice of other users. The addition of data element $e_{64}$ allows to establish communication with the user $u_{1}$. This connection is reflected by vertical arrows. Adding a new link gives the opportunity to form recommendations using collaborative filtering.

Experimental verification of the method was performed using the Online Retail dataset located in the UCI storage.

This kit is a sales log for a UK chain of gift shops. This network makes wholesale purchases. Such purchases are repeated at regular intervals. Interest in gifts may also change cyclically. As part of the experiment, a subset of 10.4 thousand records was selected that contain repeated purchases by the same users, which allows them to find temporal constraints. A subset of the data was supplemented by a record of one purchase of a new buyer.

During the experiment, the value of AUC (Area Under the Curve) was compared for the recommended list of the most popular items, as well as the recommendations obtained using temporal 
constraints. In the first case, for the dataset used, the AUC value was 0.71 , and in the second -0.72 . The increase in accuracy is small, but it should be borne in mind that a small dataset was used. In general, the experimental results indicate the influence of temporal limitations. To further improve the accuracy, it is necessary to use weighted temporal dependencies that define possible patterns of user choice.

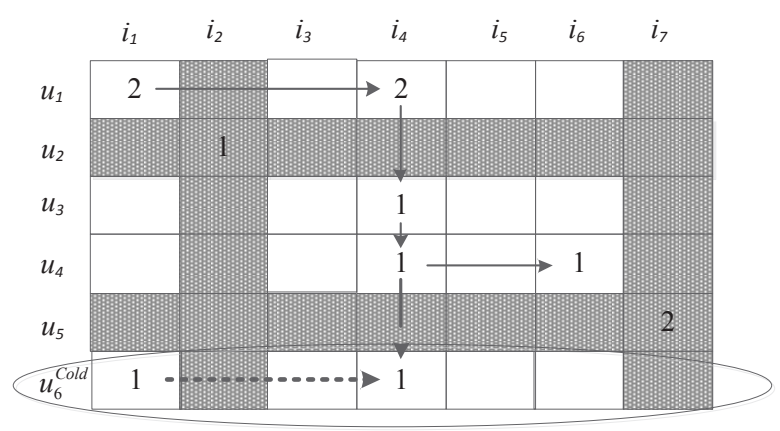

Fig. 2. Updated raw data

\section{The discussion of the results}

The result of the work is the method of forming recommendations in the situation of a cyclical cold start. This situation occurs for users who occasionally turn to the recommendation system.

The recommended list of items that are obtained as a result of the completion of the method allows personalizing the consumer choice in the conditions of incomplete data about the user of the recommendation system.

The difference of the proposed method lies in the preliminary modification of the input data of the new user, taking into account the temporal restrictions on the choice of existing consumers.

The advantage of the method is that it is possible using temporal constraints to clarify recommendations for users who access the recommendation system occasionally, at long intervals. Temporal limitations make it possible to form a connection between the choice of new and wellknown users.

The disadvantage of the method is that the temporal limitations used when forming recommendations and, as a result, the composition of the recommended list of items, largely depend on the time interval in which the initial data is selected.

The method has limitations related to the features of offline and online modes of operation of the recommendation system. The method is focused only on the online mode of operation, since it does not take into account the entire data set, as in offline mode, but only the most relevant input data, reflecting the cycle of changing user priorities.

The developed method is intended for use in the situation of a cyclic cold start of the recommendation system associated with the periodic change in user preferences. The method complements the existing approaches to building recommendations based on collaborative filtering.

\section{Conclusions}

The problem of building a list of recommended objects in the situation of a cyclic cold start characterized by a periodic change in consumer preferences is considered. This complicates the construction of recommendations for new, "cold" users. In such a situation it is necessary to consider patterns that characterize changes in consumer interests. These patterns set constraints when forming recommendations and are usually not taken into account within the framework of existing approaches.

A method for constructing recommendations under cyclic cold start conditions using temporal constraints is proposed. The method includes the steps of forming temporal constraints, supplementing data for new users based on these constraints, as well as generating recommendations using an augmented data set.

This method, in contrast to existing ones, allows using cyclical changes in the choice of consumers to personalize recommendations to new users. 
The proposed method makes it possible to increase the efficiency of forming recommendations for new "cold" users in a situation of periodic changes in their interests, taking into account temporal limitations for known users.

With further improvement of the method, it is also assumed to take into account the weighted temporal dependencies that characterize typical patterns of user choice over time. The key difference between the considered restrictions and such dependencies is that the latter do not set mandatory, but possible options for changing user preferences.

\section{References}

[1] Aggarwal, C. (2017). Recommender Systems. New York: Springer, 498.

[2] Son, L. H. (2016). Dealing with the new user cold-start problem in recommender systems: A comparative review. Information Systems, 58, 87-104. doi: http://doi.org/10.1016/j.is.2014.10.001

[3] Bernardi, L., Kamps, J., Kiseleva, J., Müller, M. (2015). The Continuous Cold Start Problem in e-Commerce Recommender Systems. 2nd Workshop on New Trends on Content-Based Recommender Systems, 30-33.

[4] Kalynychenko, O., Chalyi, S., Bodyanskiy, Y., Golian, V., Golian, N. (2013). Implementation of search mechanism for implicit dependences in process mining. 2013 IEEE 7th International Conference on Intelligent Data Acquisition and Advanced Computing Systems (IDAACS). doi: http://doi.org/10.1109/idaacs.2013.6662657

[5] Braunhofer, M. (2014). Hybrid Solution of the Cold-Start Problem in Context-Aware Recommender Systems. Lecture Notes in Computer Science, 484-489. doi: http://doi.org/10.1007/978-3-319-08786-3_44

[6] Lika, B., Kolomvatsos, K., Hadjiefthymiades, S. (2014). Facing the cold start problem in recommender systems. Expert Systems with Applications, 41 (4), 2065-2073. doi: http://doi.org/10.1016/j.eswa.2013.09.005

[7] Koren, Y. (2009). Collaborative Filtering with Temporal Dynamics. ACM SIGKDD International Conference on Knowledge Discovery and Data Mining, 447-456. doi: http://doi.org/10.1145/1557019.1557072

[8] Xiang, L., Yuan, Q., Zhao, S., Chen, L., Zhang, X., Yang, Q., Sun, J. (2010). Temporal Recommendation on Graphs via Longand Short-term Prefence Fusion. KDD'10 of the 16th ACM SIGKDD International Conference on Knowledge Discovery and Data Mining, 723-732. doi: http://doi.org/10.1145/1835804.1835896

[9] Elahi, M., Ricci, F., Rubens, N. (2016). A survey of active learning in collaborative filtering recommender systems. Computer Science Review, 20, 29-50. doi: http://doi.org/10.1016/j.cosrev.2016.05.002

[10] Chalyi, S., Pribylnova, I. (2019). The Method of Constructing Recommendations Online on the Temporal Dynamics of User Interests Using Multilayer Graph. EUREKA: Physics and Engineering, 3, 13-19. doi: http://doi.org/10.21303/2461-4262. 2019.00894

[11] Luo, C., Cai, X., Chowdhury, N. (2014). Self-training Temporal Dynamic Collaborative Filtering. Lecture Notes in Computer Science, 461-472. doi: http://doi.org/10.1007/978-3-319-06608-0_38

[12] Zhu, Y., Lin, J., He, S., Wang, B., Guan, Z., Liu, H., Cai, D. (2019). Addressing the Item Cold-start Problem by Attribute-driven Active Learning. IEEE Transactions on Knowledge and Data Engineering. doi: http://doi.org/10.1109/tkde.2019.2891530

[13] Levykin, V., Chala, O. (2018). Development of a method for the probabilistic inference of sequences of a business process activities to support the business process management. Eastern-European Journal of Enterprise Technologies, 5 (3 (95)), 16-24. doi: http://doi.org/10.15587/1729-4061.2018.142664

[14] Levykin, V., Chala, O. (2018). Method of Determining Weights of Temporal Rules in Markov Logic Network for Building Knowledge Base in Information Control Systems. EUREKA: Physics and Engineering, 5, 3-10. doi: http://doi.org/10.21303/24614262.2018.00713

[15] Chalyi, S., Levykin, I., Petrychenko, A., Bogatov, I. (2018). Causality-based model checking in business process management tasks. IEEE 9th International Conference on Dependable Systems, Services and Technologies DESSERT'2018. Kyiv, 478 -483. doi: http://doi.org/10.1109/dessert.2018.8409176

[16] Baltrunas, L., Ludwig, B., Ricci, F. (2011). Matrix factorization techniques for context aware recommendation. Proceedings of the Fifth ACM Conference on Recommender Systems, 301-304. doi: http://doi.org/10.1145/2043932.2043988 